\title{
Erratum to: Immunohistochemical molecular expression profile of metastatic brain tumor for potent personalized medicine
}

\author{
Yasutaka Kato $\cdot$ Hiroshi Nishihara $\cdot$ Sayaka Yuzawa \\ Hiromi Mohri · Hiromi Kanno • Yutaka Hatanaka • \\ Taichi Kimura $\cdot$ Mishie Tanino $\cdot$ Shinya Tanaka
}

Published online: 19 March 2013

(C) The Japan Society of Brain Tumor Pathology 2013

\section{Erratum to: Brain Tumor Pathol}

DOI 10.1007/s10014-012-0124-y

Unfortunately, the case numbers of EGFR (del) in rows 2 and 3 of Table 3 have been incorrectly published in the original publication. Table 3 should be correctly shown as given below:

The online version of the original article can be found under doi:10.1007/s10014-012-0124-y.

Y. Kato $\cdot$ S. Yuzawa $\cdot$ H. Kanno $\cdot$ T. Kimura $\cdot$ M. Tanino .

S. Tanaka

Department of Cancer Pathology, Hokkaido University Graduate

School of Medicine, Sapporo, Japan

H. Nishihara ( $\square)$

Department of Translational Pathology, Hokkaido University

Graduate School of Medicine, North 15, West 7,

Kita-Ku, Sapporo 060-8638, Japan

e-mail: hnishihara@s5.dion.ne.jp;

hnishihara@med.hokudai.ac.jp

H. Mohri

Laboratory of Oncology, Hokuto Hospital, Obihiro, Japan

Y. Hatanaka

Department of Surgical Pathology,

Hokkaido University Hospital, Sapporo, Japan 


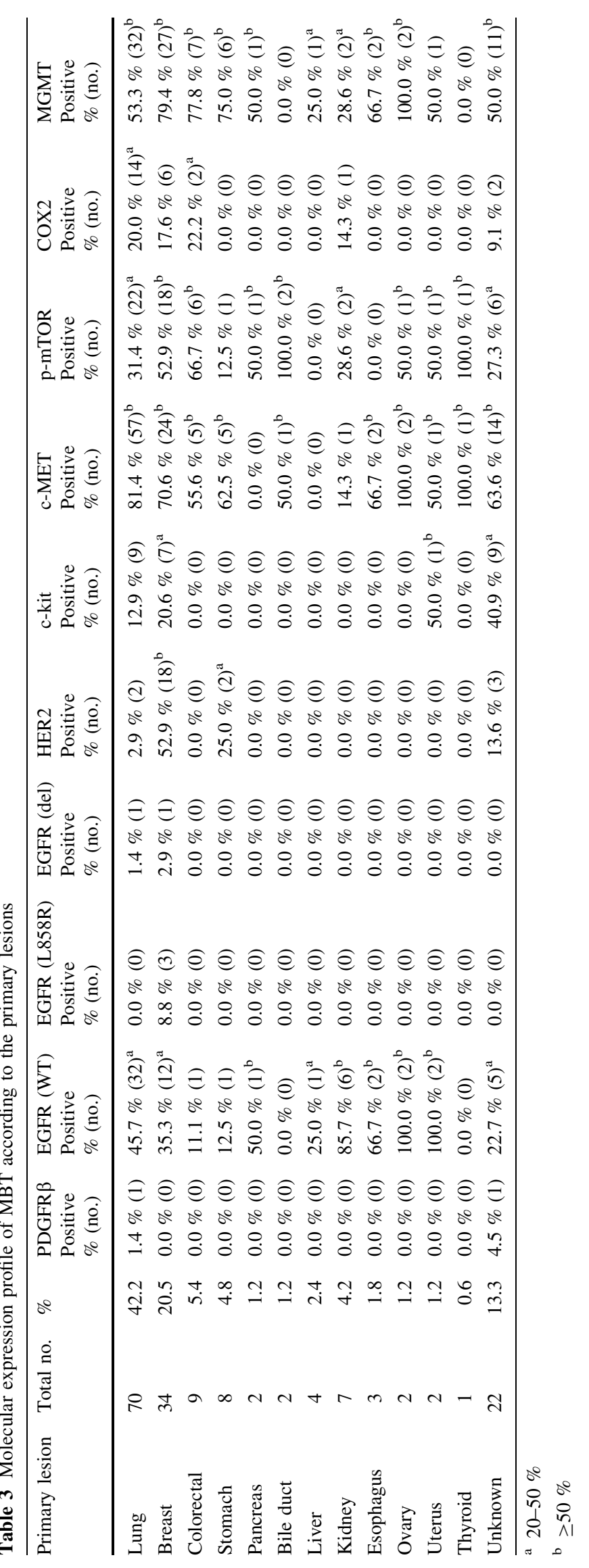

